Published in final edited form as:

Aphasiology. 2017 ; 31(4): 447-464. doi:10.1080/02687038.2016.1271105.

\title{
Corpus-Based Transitivity Biases in Individuals with Aphasia
}

\author{
Jennifer DiLallo ${ }^{1}$, Heidi Mettler ${ }^{1}$, and Gayle DeDe ${ }^{2}$ \\ ${ }^{1}$ Department of Speech, Language, and Hearing Sciences; University of Arizona, Tucson, AZ \\ ${ }^{2}$ Department of Communication Sciences and Disorders, Temple University, Philadelphia, PA
}

\begin{abstract}
Background-This study investigated whether individuals with aphasia (IWA) retain verb biases in expressive language. Verb biases refer to the likelihood that a given verb will occur in different sentence structures. We focused on the likelihood of verbs occurring in transitive and intransitive structures.
\end{abstract}

\begin{abstract}
Aims-The main goal of this study was to determine whether IWA and controls show similar verb biases or whether IWA show a preference for transitive or intransitive structures that supersedes individual verb biases. We also investigated whether IWA show a preference for intransitively or transitively biased verbs, whether verb biases differ as a function of aphasia type, and how verb bias affects errors in IWA's speech production.
\end{abstract}

Methods-The current study analyzed 236 transcribed interviews of IWA from AphasiaBank. All uses of 54 verbs were coded based on the sentence structure and the presence of errors. We report data from 11 transitively biased and 11 intransitively biased verbs.

Outcomes and Results-IWA's transitivity biases were indistinguishable from controls' biases. In addition, IWA produced more intransitively biased verbs than transitively biased verbs overall. In ungrammatical productions, IWA's error rates were higher in sentence structures that conflicted with verb bias and highest when an intransitively biased verb was attempted in a transitive structure.

Main Conclusions-These findings indicate that IWA are sensitive to verb bias and verb complexity within expressive language. The effects are consistent with previous literature concerning IWA's sensitivity to verb bias in receptive language tasks and to verb complexity in verb retrieval tasks.

\section{Keywords}

aphasia; transitivity; verb bias; expressive language; AphasiaBank

\section{Introduction}

A large body of literature suggests that individuals with aphasia (IWA) have more difficulty retrieving verbs than nouns in confrontation naming tasks and in spontaneous speech (e.g.,

Contact Author: Gayle DeDe, Department of Communication Sciences and Disorders Temple University, Weiss Hall, Room 1101701 N $13^{\text {th }}$ Street, Philadelphia, PA 19122, Phone: 215-204-2453, gayle.dede@temple.edu. 
Basso, Razzano, Faglioni, \& Zanobio, 1990; Berndt, Haendiges, Mitchum, \& Sandson, 1997a, 1997b; Kim \& Thompson, 2000; Lee \& Thompson, 2015; Miceli, Silveri, Villa, Caramazza, 1984). This observation has led to a lot of interest regarding how IWA process verbs, and what sorts of psycholinguistic variables contribute to this noun-verb dissociation. The current study focused on how IWA use transitively and intransitively biased verbs in spontaneous speech samples.

Verb argument structure characterizes how many arguments (subject, direct object, indirect object) are present in a verb phrase. Most verbs can occur in more than one argument structure. For example, an optionally transitive verb such as eat can occur in both transitive and intransitive sentence frames, as seen in examples (1) and (2). Verbs differ from one another in the frequency with which they occur in different sentence structures. For example, eat occurs most frequently in transitive structures, whereas dance occurs most frequently in intransitive structures (example 3). However, dance can also occur in transitive structures (example 4). Here, we focus on the frequency with which verbs are or are not followed by a direct object. We will refer to this as transitivity bias.

1. The friends eat [D.O. salsa] [ $A_{d V}$ happily].

2. The friends eat [ $A_{V}$ happily].

3. The friends dance.

4. The friends dance [D.O. the salsa].

There are many structures other than transitive and intransitive frames (e.g. sentential complements, passives, and infinitives), and so there are multiple kinds of verb biases. For example, know is often followed by a sentential complement (e.g. "I know [S.C. that this is a sentential complement]"). However, simple transitive and intransitive sentences are the most commonly used structures in the English language (Roland, Dick, \& Elman, 2007). Thus, we will focus primarily on the "transitivity bias" of verbs that occur in transitive and intransitive sentence structures.

Transitivity biases affect both receptive and expressive language. In order to establish verb biases, researchers generally calculate the frequency with which a population produces a verb in different structures across samples of written or spoken language. We are then able to evaluate how verb biases influence language processing. Until now, no studies have described whether IWA show the same verb biases as controls in expressive language. However, previous studies have compared IWA 's and controls' sensitivity to normative verb biases in comprehension tasks, and we can use that literature to develop predictions about the nature of IWA's verb biases in expressive language.

The study of verb biases has largely been based on studies of non-brain-damaged individuals. Verb biases have been determined through two main methods: sentence elicitation and corpus analysis. In elicitation studies, participants are provided with verbs and asked to generate sentences with each verb. In corpus studies, verbs are coded based on their usages in large language samples (e.g., taken from newspapers or structured interviews). The advantage of language corpora is that they represent more naturalistic language productions. However, corpora samples may vary by sample context (e.g., an 
academic journal vs. a radio station), population size, and coding decisions (e.g., which verbs to code).

For this reason, cross-corpora studies like Gahl, Jurafsky, and Roland (2004) are especially helpful in understanding replicable trends across corpora. Their study focused on verb subcategorization frequencies (of which transitivity bias is one type) in two separate corpora (the COMLEX database and British National Corpus). Gahl and colleagues developed a coding schema to label a number of sentential frames, including simple transitive and simple intransitive sentences. Then, they compared their verb biases to those of five elicitationbased studies. The team evaluated how various coding decisions affected verb biases - like the effect of using relative proportions of use or an absolute criterion (e.g. two thirds of all uses) to establish verb bias. For transitivity, the research team compared direct object (DO) to sentential complement (SC) biased verbs, and found that 167 of 281 verbs changed bias based on the analysis. Their study suggested that absolute criterions overlook smaller, though significant, transitive biases. Thus, they recommended use of relative proportions (i.e., the relative frequency with which verbs occur in different structural frames).

There is evidence that these sorts of verb biases influence language comprehension in nonbrain-damaged populations (e.g., Garnsey, Pearlmutter, Myers, \& Lotocky, 1997; Staub 2007). Individuals with aphasia are also sensitive to transitivity biases during language comprehension (DeDe, 2012; 2013a, 2013b; Gahl, 2002). Gahl (2002) performed a comprehension study of simple transitive, intransitive, and passive sentences with controls $(\mathrm{n}=5)$ and IWA $(\mathrm{n}=17)$. Participants listened to sentences with transitively and intransitively biased verbs in structures that either supported or violated the verb's biases (i.e., in matched and mismatched conditions, respectively). Both IWA and controls made more errors in the mismatched condition, though IWA made proportionally more errors than controls. These results were considered to be consistent with the Lexical Bias Hypothesis, which suggests that IWA are more sensitive than non-brain-damaged individuals to violations of verb bias.

DeDe (2012; 2013a, 2013b) showed that IWA are sensitive to verb biases during real time sentence processing. In one study, IWA and controls participated in a self-paced reading paradigm with sentences featuring sentential complements (DeDe, 2013a). Main verbs were biased to take either a direct object or sentential complement. Each sentential frame occurred with and without the complementizer that. Sentences without the complementizer were temporarily structurally ambiguous, meaning that there were two possible interpretations of the noun phrase following the main verb. For example, in a sentence fragment such as "The friends knew the girl...," the noun phrase the girl could be the object of the verb knew or the subject of a sentential complement (as in, "The friends knew the girl would eat all the salsa."). IWA's group performance did not significantly differ from controls in relation to sensitivity to verb bias. However, analyses of individual data showed that seven of the nine IWA demonstrated a greater sensitivity to verb bias than structural ambiguity when compared to controls. That is, verb bias had a greater effect on reading times than the presence or absence of the complementizer. These findings suggest that IWA access verb bias to navigate sentence comprehension, and rely more on this lexical feature than control populations. 
Differences in sensitivity to verb bias in sentence comprehension may reflect underlying changes in the representation of verb bias in IWA. That is, IWA may develop stronger verb biases, leading to exaggerated effects of verb bias in both receptive and expressive language. Alternatively, verb biases could be similar in IWA and non-brain-damaged populations. In this scenario, apparent differences in comprehension studies could reflect different weighting of cues (e.g., verb bias vs. complementizer) rather than differences in the cues themselves. Until recently, there was no corpus available to analyze the productive biases of IWA. Instead, researchers recorded spontaneous speech samples from small groups of IWA. Previous studies of these groups have considered effects of argument structure and verb phrase complexity on IWA's speech, but no study has directly examined verb bias in IWA's expressive language.

Saffran, Berndt, and Schwartz (1989) elicited speech from ten IWA and five controls using the Cinderella story (a common narrative in language research). They coded the speech samples in terms of accuracy (how well-formed sentences were) and elaboration of noun phrase and verb phrase constituents within a sentence. The authors reported that most of the 52 ill-formed sentences included morphological errors. Only six ill-formed utterances involved omission of an argument. These results suggest that IWA infrequently omit arguments in sentences, but they do not address the issue of verb bias.

Webster, Franklin, and Howard (2007) found a somewhat different pattern of results in their analysis of spontaneous speech samples with IWA. Speech samples were coded using Whitworth's (1995) schema of thematic structure, which allowed them to examine the omission of obligatory arguments in two and three argument structures. As a group, IWA produced more sentences with fewer or undetermined thematic structures than controls. The results showed that IWA were more likely to omit obligatory arguments than the control group, but it was not clear whether they made more errors on certain sentence types.

Taken together, these studies reveal that IWA may sometimes omit arguments. However, neither study considered verb bias, so we do not know whether IWA show similar verb biases as controls in expressive language tasks. The present study addressed verb bias, specifically transitivity bias, using data from AphasiaBank, which is a corpus database that includes speech samples of IWA from around the United States (MacWhinney, Fromm, Forbes, \& Holland, 2011). Participants with different aphasia types generated spontaneous speech samples in response to a range of prompts.

Our primary research question was whether IWA are sensitive to transitivity bias in their spontaneous speech. Based on the comprehension data reviewed above (e.g., DeDe, 2013a, 2013b; Gahl, 2002), we expected IWA to be sensitive to transitivity biases in speech production. Further, IWA's performance on the production tasks can help adjudicate different accounts of why they show greater reliance on verb bias in comprehension tasks. One possibility is that IWA's reliance on verb biases in comprehension reflects exaggerated, or stronger, underlying biases than controls. On this account, IWA should also show stronger verb biases than controls in production tasks. A second possibility is that IWA show greater reliance on verb bias in comprehension tasks because they weight cues differently than 
controls. On this account, IWA and controls would have similar underlying representation of verb bias, resulting in similar verb biases for the two groups in production tasks.

An alternative hypothesis regarding our first research question is that IWA rely less on verb bias than non-brain-damaged populations in speech production. On this account, IWA's expressive language might show a preference for transitive or intransitive structures that outweighs verb bias. This pattern could emerge if IWA favored transitive structures, which might occur because transitive sentences are produced more frequently in English (Gahl et al., 2004; Roland et al., 2007). Alternatively, IWA might favor intransitive structures because they only require one argument (i.e., the sentential subject).

Next, we asked whether IWA would favor intransitively or transitively biased verbs, regardless of sentence structure. There is evidence that IWA have an easier time retrieving intransitively biased than transitively biased verbs in elicitation tasks. Kim and Thompson (2000) examined IWA's verb naming ability as a function of argument complexity. In a confrontation naming task, IWA retrieved intransitively biased verbs more accurately than transitively biased verbs. This type of finding led to our second research question, which was whether IWA produce more intransitively than transitively biased verbs.

Our third research question was whether IWA would make more errors when producing sentences with a mismatch between transitivity bias and sentence structure (e.g., transitively biased verbs in an intransitive structure). Given that IWA make more comprehension errors when there is a mismatch between verb bias and sentence structure, we expected higher error rates when IWA produce mismatched structures.

Finally, we considered whether people with Broca's and Wernicke's aphasia demonstrate qualitatively different patterns of verb bias. There is a large literature focused on 11 the noun-verb dissociation in people with aphasia (e.g., Basso et al., 1990; Berndt et al., 1997a, 1997b; Kim \& Thompson, 2000; Lee \& Thompson, 2015; Miceli et al., 1984). Some authors report that individuals with Broca's aphasia have more difficulty producing verbs than nouns and that individuals with anomic - or other fluent aphasias - do not show this dissociation between nouns and verbs. A full review of this literature is beyond the scope of this paper. Here, the critical question is whether there is an effect of aphasia type on transitivity biases. Studies of sentence comprehension (e.g., DeDe, 2013a, 2013b; Gahl, 2002) have not found significant differences in sensitivity to verb bias in individuals with fluent and non-fluent aphasia. Based on these studies, we predicted that aphasia type would not influence sensitivity to verb bias in IWA.

In summary, this study focused on the following questions: (1) How do IWA's transitivity biases compare to control populations? (2) Do IWA show an overall preference for intransitive verbs? (3) Do IWA make more errors when producing utterances in which the sentence structure conflicts with the verb's bias? (4) Do individuals with Broca's or Wernicke's aphasia show a different pattern than the larger group of IWA? To address these questions, we examined IWA's intransitive and transitive productions of a set of intransitively biased and transitively biased verbs. 


\section{Methods}

\section{Participants}

270 morphologically and grammatically transcribed interviews of participants with aphasia were analyzed from the AphasiaBank database using CHAT and CLAN transcription programs (MacWhinney, 2000). Of these, 34 were omitted because they were not monolingual English speakers. The remaining 236 participants were native English speakers between the ages 25 and 90 years old. According to the Boston classification system, 73 participants were classified with anomic aphasia, 73 with Broca's, 26 with conduction, 14 with global, 15 with Wernicke's, and 7 with transcortical motor. The remaining 28 participants' aphasia types were unidentified, non-classifiable, or other. We examined the corpus to determine whether one participant contributed a disproportionate amount of data to the analysis. Though there was some variability, no participant contributed more than $3 \%$ of the data to the verb corpus, with an average contribution of .4\%.

\section{Materials}

Participants in AphasiaBank performed the same tasks in a video-recorded session: 1) sharing their aphasia story and coping experiences, 2) talking about an important life event, 3) picture description, 4) Cinderella story, and 5) describing how to make a peanut butter sandwich. Instructions and examples of protocol are available on the AphasiaBank website. Interviews of IWA were downloaded October 31, 2014.

We also coded data from 41 non-brain-damaged control participants from AphasiaBank who performed the same protocol as IWA. One modification is that these participants shared any illness story for Task 1. These individuals ranged between 23-89 years old and were native speakers of English. Interviews of controls were downloaded April 4, 2015.

Verbs of interest-We coded the sentential structures of all productions of 54 verbs throughout AphasiaBank. The verbs were selected in part through an informal review of the transcripts in AphasiaBank. First, we reviewed all verb productions from 14 participants to identify verbs that were likely to be elicited by the corpus' language tasks. Our goal was to identify a set of verbs that would occur frequently enough to support conclusions about verb bias. We selected 54 common verbs, which showed biases toward transitive, intransitive, passive, and sentential complement structures based on Gahl et al.'s (2004) data. This paper focuses on a subset of those verbs: 11 transitively biased verbs and 11 intransitively biased verbs. Verbs were classified as transitively biased if they occurred in transitive frames at least 25\% of the time in Gahl et al.'s (2004) data and if they occurred in transitive frames at least twice as often in transitive than intransitive frames. Intransitively biased verbs occurred in intransitive frames at least $25 \%$ of the time and occurred in intransitive frames at least twice as often in intransitive versus transitive frames. ${ }^{1}$ The 22 verbs were matched for frequency and number of syllables (Table 1). Only the data from this subset will be considered in the following analyses.

\footnotetext{
${ }^{1}$ The verb start was coded as an intransitively biased verb due to a calculation error, though it did not meet the criteria in Gahl et al.'s (2004) data. The current data were analyzed with and without the verb start. The results did not change in the two sets of analyses. Results reported here include the verb start so as to maintain the balance between the sets.
} 
Coding-The verb-coding scheme was based on the one described by Gahl et al. (2004). We made minor adjustments to accommodate the challenges of coding speech from a disordered population. Example verb codes from this study are shown in Table 2, and they are described in supplementary materials associated with this manuscript. With respect to the present paper, an important consideration was how we differentiated particles and prepositions. The presence of a particle could determine whether a sentence frame was coded as transitive or intransitive. For instance, "look up a definition" is a verb + particle construction while "look up a tree" is a verb + preposition construction. Critically, look would be coded as transitive when up is viewed as a particle, or intransitive when up is viewed as a preposition. As described in the coding scheme, we employed a series of "prepicle tests" based on those listed in Darwin and Gray (1999) to distinguish the two categories. ${ }^{2}$ Particle constructions were collapsed within the verb counts of the selected 22 verbs. Gahl et al. (2004) examined how inclusion of particle constructions influenced verb bias in 280 verbs and found that particles only influenced verb bias classifications for $4 \%$ of the verbs they studied. None of the verbs that were affected by inclusion of particles in Gahl et al. (2004) were examined in the present study.

A second important coding decision was related to ungrammatical utterances. For example, "She danced the prince" could be treated as a transitive use of dance or an intransitive use with an omitted function word. We made the decision to always code on the surface level. Even though a participant's intended utterance sometimes seemed apparent, especially with simple omissions, coders only labeled the participant's actual production. That way, we avoided imposing grammaticality on the speech of a participant and imposing our own verb biases on the participants. We return to the issue of how this might have affected our results in the discussion.

Two coders with training in linguistics (first and second author) applied the coding scheme to the verb tokens. Inter- and intra-rater reliability was measured through Cohen's Kappa $(\mathrm{K})$, a statistic that measures percent agreement due to chance. Acceptable reliability levels were based on Gahl et al. (2004)'s criteria of a Kappa value greater than 0.80.

We also developed an error-coding scheme to account for non-grammatical speech. We were principally concerned with errors that affected the verb phrase. Error codes with examples are provided in Table 3, and the error codes are described in detail in the supplementary materials. Here, we provide a brief overview. The coding scheme characterized errors based on position (before, on, or following the verb) and type, when possible (e.g., phonological, semantic, inflectional). Phonological and semantic paraphasias on the target verb itself were based on transcripts downloaded from AphasiaBank. Some errors were unambiguous, often a lexical error or an omission of a function word. For instance, utterances such as "He drove her the ball" were treated as omissions of a function word (note: the example sentence is referring to Cinderella's ball). To be classified as such, both coders had to agree that omission of a function word was the only likely interpretation. However, most errors were more ambiguous. These were classified more generally as error after verb (eav) or error before verb (ebv). Verb phrases could be associated with multiple error codes. For example,

\footnotetext{
${ }^{2}$ We used the term "prepicle" to refer to a word before classifying it as preposition or particle.
} 
"because she see of something..." contains both an inflectional error on the verb ("she see") and an error after the verb ("see of something"). Two coders reviewed all error coding decisions ( $2^{\text {nd }}$ and $3^{\text {rd }}$ author). Coding questions were resolved through group discussion and consensus.

\section{Results}

\section{Reliability}

Inter-rater reliability was evaluated at the beginning and end of data coding. Initially, the coders $\left(1^{\text {st }}\right.$ and $2^{\text {nd }}$ author) coded five transcripts and had an inter-rater reliability of $\mathrm{K}=$ 0.77. The research team met to discuss coding conflicts, focusing on adaptations for aphasic speech, and reached coding decisions through consensus. The second round of pre-coding (again with five transcripts) had an inter-rater reliability of $K=0.83$.

At this point, the coders began coding the remaining transcripts separately. Throughout the coding process, the coders marked instances of uncertainty to discuss in lab meetings. Coding decisions for these utterances were decided on a consensus-basis with the third author. In order to ensure that there was not significant procedural drift in the coding, interrater reliability was also checked after all transcripts were coded. For this calculation, each coder recoded approximately $8 \%$ of the other coder's files (based on total number of target verb uses). Inter-rater reliability after coding was $\mathrm{K}=0.86$. Finally, each coder re-coded $5 \%$ of their own files to evaluate intra-rater reliability. Both coders were above the acceptability criteria $(\mathrm{K}=0.92$ and $\mathrm{K}=0.88)$.

\section{Verb Biases}

For each verb, the proportion of transitive or intransitive uses was calculated by dividing the number of transitive or intransitive uses by the total number of occurrences for the verb. This proportion was the dependent variable in 2 (group: IWA vs. controls) by 2 (sentence structure: transitive vs. intransitive) repeated measure ANOVAs, separately for transitively biased verbs and intransitively biased verbs. Figure 1 presents how IWA and controls used transitively biased verbs in the AphasiaBank sample. Figure 2 presents how IWA and controls used the intransitively biased verbs. The remaining productions in the figures represent a variety of structures including infinitives, NP-complements, and sentential complements. These structures were not included in any of the analyses. The segments of interest are the white pie pieces, which denote transitive uses of transitively and intransitively biased verbs, and the black pie pieces, which denote intransitive uses of transitively and intransitively biased verbs.

Transitively Biased Verbs-IWA used transitively biased verbs in transitive structures an average of $43.9 \%$ of the time (e.g., "The friends eat salsa"; white segment in figure 1). They used transitively biased verbs in intransitive structures about $14.7 \%$ of the time verbs (eg. "The friends eat"; black segment in figure 1). Controls used transitively biased verbs in transitive structures $50.7 \%$ of the time and in intransitive structures $7.1 \%$ of the time. The main effect of sentence structure was significant, $\mathrm{F} 1(1,10)=18.5, \mathrm{p}<.001$, due to an increased proportion of transitive uses for transitively biased verbs, for both groups. Neither 
the main effect of group nor the interaction of group and sentence structure was significant (F's $\leq 1.4)$.

Intransitively Biased Verbs-Both groups produced intransitively biased verbs in intransitive structures more frequently than transitive structures. In IWA, intransitively biased verbs were used in intransitive structures $55.5 \%$ of the time (eg. "The friends dance", black segment in figure 2), and in transitive structures $3.7 \%$ of the time (e.g., "The friends dance salsa"; white segment in figure 2). Controls similarly produced intransitively biased verbs in intransitive structures more often $(55.1 \%)$ than in transitive structures $(1.3 \%)$. The main effect of sentence structure was significant, $F(1,10)=27.6, p<.001$. The main effect of group and the interaction of group by sentence structure were not significant ( $\left.F^{\prime} s \leq 1\right)$.

\section{Intransitive or Transitive Verb Preference}

It is possible that IWA show a preference for intransitively or transitively biased verbs when speaking, even while maintaining the same verb biases as controls. To address this question, we analyzed the total number of times that IWA and controls used the transitively and intransitively biased verbs, regardless of sentential structure. These data were analyzed using chi-square rather than ANOVA because we were counting how many times each verb type was used and asking whether there was a statistical association between the verb type (transitively or intransitively biased) and the group (IWA vs. control).

As Figure 3 shows, both groups produced more intransitively than transitively biased verbs (regardless of sentential structure). However, the preference for producing intransitively biased verbs was larger in IWA than controls, $X^{2}(1)=27.8, p<.001$. IWA produced 2174 intransitively biased verbs (62.5\%) compared to 1307 transitively biased verbs (37.5\%). Controls produced 752 intransitively biased verbs $(54.2 \%)$ and 635 transitively biased verbs $(45.8 \%)$.

\section{Error Rates}

These analyses examined whether IWA produced more errors when there was a mismatch between transitivity bias and sentence structure (e.g., transitively biased verbs in intransitive structures and intransitively biased verbs in transitive structures) compared to when transitivity bias matched sentence structure (e.g., transitively biased verbs in transitive structures and intransitively biased verbs in intransitive structures). Controls were not included in these analyses. Error rates were analyzed in 2 (Verb bias) $\times 2$ (Sentence Structure) ANOVA. The proportion of utterances with errors is presented in Figure 4. The main effect of sentence structure was not significant, $\mathrm{F}(1,20)=3.9, \mathrm{p}=.06$. There was a marginal main effect of verb bias, $\mathrm{F}(1,20)=4.3, \mathrm{p}=.052$. IWA made more errors in the mismatched than matched condition overall. The interaction of verb bias and structure was significant, $F(1,20)=11.6, p<0.01$. IWA's errors on transitive structures with an intransitively biased verb (i.e., the mismatched condition) accounted for $50.5 \%$ of all errors, while intransitive structures with an intransitively biased verb (i.e., the matched condition) accounted for $9.9 \%$ of all errors. Error rates for transitively biased verbs followed the same pattern. IWA committed $18.3 \%$ of all errors for transitively biased verb in intransitive structures (mismatch condition) and $7.6 \%$ for transitively biased verbs in transitive structures 
(match condition). Thus, IWA made more errors when using intransitively biased verbs in transitive structures than when using transitively biased verbs in intransitive structures.

\section{Aphasia Type}

Some authors have argued that individuals with agrammatic Broca's aphasia have more impaired verb retrieval than other aphasia types. To investigate this issue, we examined whether the subset of individuals with Broca's aphasia showed a different pattern of transitivity biases than the group as a whole using ANOVA. There was a significant effect of verb bias for both the proportion of transitive $(\mathrm{F} 1(1,20)=19.4 ; \mathrm{p}<0.001)$ and intransitive $(\mathrm{F} 1(1,20)=12.9 ; \mathrm{p}<0.002)$ uses. In transitive uses, transitively biased verbs accounted for $46.8 \%$ of transitive productions, as compared to $3.7 \%$ of transitive structures produced with intransitively biased verbs. In intransitive uses, intransitively biased verbs accounted for $70.8 \%$ of productions, and transitively biased verbs accounted for $26.5 \%$. These results suggest that transitivity biases for individuals with Broca's aphasia were similar to both the controls and the larger group of IWA.

We also considered the subset of participants with Wernicke's aphasia. None of the participants with Wernicke's aphasia produced the verbs determine, disappear, or think. Nonetheless, aphasia type did not influence transitivity bias. The effect of verb bias was significant for both proportion of transitive $(\mathrm{F} 1(1,17)=57.4, \mathrm{p}<.001)$ and intransitive uses $(\mathrm{F} 1(1,17)=28.9, \mathrm{p}<.001)$. Participants produced transitive structures with transitively biased verbs $50.6 \%$ of the time and with intransitively biased verbs $2.5 \%$ of the time. Conversely, in intransitive structures, participants produced intransitively biased verbs $79.4 \%$ of the time and transitively biased verbs $20.2 \%$ of the time.

\section{Discussion}

The results show that IWA preserve sensitivity to verb bias in their spontaneous speech, at least for the transitively and intransitively biased verbs examined in this study. Both controls and IWA produced intransitively biased verbs more often in intransitive than transitive structures, and transitively biased verbs more often in transitive than intransitive structures. Our findings are consistent with Gahl et al. (2004)'s transitivity biases, which were based on a much larger corpus of data. The results are also consistent with previous studies (DeDe, 2013a, 2013b; Gahl, 2002), which demonstrated that IWA are sensitive to verb biases in sentence comprehension.

With respect to our primary research question, the results suggest that IWA are sensitive to transitivity biases-but that verb biases are not stronger in IWA than controls and that preferences for a particular structure (transitive or intransitive) do not outweigh verb biases. Recall that, in reading comprehension tasks, IWA relied more on verb bias than controls (DeDe, 2012; 2013a, 2013b). The reading data could reflect an underlying difference in the verb biases themselves or a difference in how IWA use verb bias during comprehension. In the present study, IWA's transitivity biases were statistically indistinguishable from controls' biases. These results suggest that IWA's performance in comprehension studies resulted from a greater dependency on verb bias than controls and not from underlying stronger verb biases. 
More holistically, IWA exhibited an overall preference for intransitively biased verbs. They produced more intransitively biased verbs than transitively biased verbs - regardless of sentential structure - and this difference (though in the same direction) was significantly greater in IWA than controls. This preference is consistent with previous studies showing that IWA named obligatory intransitive verbs and optionally transitive verbs more accurately than obligatory transitive verbs (Kim \& Thompson, 2000). Kim and Thompson (2000) suggested that intransitive verb argument structures are less complex than transitive ones. In the present study, lower complexity may denote lower cognitive costs in sentence production. This could occur because participants do not have to retrieve an additional argument (i.e. the direct object), which would account for the preponderance of intransitive uses of intransitively biased verbs. However, we found that intransitively biased verbs were used more frequently regardless of sentence structure, suggesting that retrieval of the verb itself might be less demanding regardless of the sentence structure. Further research is needed to investigate these claims.

Regarding our third research question, there was evidence that both the preference for intransitively biased verbs and transitivity biases affected IWA's error rates. IWA made more errors on utterances with mismatched biases (i.e., transitively biased verbs in intransitive sentences and vice versa) than utterances with matched biases (i.e., transitively biased verbs in transitive sentences and intransitively biased verbs in intransitive sentences). Further, of the two possible mismatched conditions, IWA made more errors when attempting to produce intransitively biased verbs in transitive structures than they did when trying to produce transitively biased verbs in intransitive structures. That is, producing the "preferred" verb type (i.e., intransitively biased verbs) in a structure that conflicted with the verb type's bias was associated with higher error rates than violations of the less preferred verb type. Thus, both verb bias and verb complexity influence the grammaticality of IWA's speech production. The implication is that utterances that violate both transitivity bias and the preference for intransitively biased verbs are associated with higher cognitive costs for IWA.

DeDe (2013a) reported an apparently conflicting pattern of results in sentence comprehension. She found that IWA had the most difficulty processing transitively biased verbs in intransitive structures. Although these results appear to be inconsistent with the present study, we do not think they are. DeDe (2013) argued that IWA showed a preference for transitive structures because transitive (SVO) structures are the most frequent in English corpus data. In the context of comprehension, transitive structures may be less cognitively demanding than intransitive structures because they are more frequent and because the arguments need to be recognized, but not retrieved. However, intransitively biased verbs may be less cognitively demanding in the context of production because only one argument needs to be retrieved. For these reasons, transitively biased verbs might be more cognitively demanding in the context of production. This distinction between comprehension and production may account for the apparently conflicting results.

A separate consideration is that our coding conventions may have influenced the results of the error analyses. We coded verbs based on actual productions rather than inferring what the IWA intended to say (i.e., the surface level). We did not want to make assumptions about what the IWA "intended to say." An example of how this could affect the error analyses can 
be seen in simple omissions of function words. IWA sometimes omit function words, so utterances like (5) could be interpreted as an intransitive structure with a missing preposition (e.g. with). However, (5) is transitive on the surface level and was coded as such. For this reason, our coding scheme could have inflated the error rates of intransitively biased verbs in transitive structures.

\section{5. *She danced the prince.}

To address this concern, we reviewed all the error codes and found that only 9 of 356 errors (2.5\%) were judged to both: 1 ) be unambiguously due to omission of a function word, and 2) to change transitivity with the insertion of a function word. Given these results, it seems unlikely that the high error rates surrounding intransitively biased verbs in transitive structures are solely a product of the coding system. This supports the claim that IWA have particular difficulty producing intransitively biased verbs in transitive structures.

We also investigated whether individuals with Broca's or Wernicke's aphasia showed different patterns of transitivity bias than the group as a whole or controls. The results showed that individuals with both aphasia types showed the same patterns of verb usage as those observed in the full sample of IWA and controls. Thus, even if verb retrieval ability is differentially affected by aphasia type, the underlying transitivity biases associated with the verbs do not seem to be affected.

One potential question is whether the speech elicitation tasks used in Aphasia Bank influenced the results. We do not think so, for several reasons. First, the samples come from a range of elicitation tasks, including personal narrative, procedural narrative, story retell, and picture description. Second, the transitivity biases observed in the present study are very similar to those reported in Gahl et al.'s (2004) corpus study. The similar results validate the results of the present study, since controls completing a very similar set of elicitation tasks as the IWA showed the same pattern of results as was seen in a much larger corpus of controls.

Another potential concern is that the verbs examined in the present study influenced the results. Some of the verbs in the present study occurred more frequently in a sentence frame other than transitive or intransitive structures. For example, think took a sentential complement in $44 \%$ of the utterances in the Gahl et al. (2004) corpora. Another issue is that the intransitive verbs comprised both unergative (e.g., dance) and unaccusative (e.g., grow) tokens. Our main focus was on the relative distribution of transitive and intransitive uses of the verbs in the participant groups. For this reason, it is unclear how differences in the strength of the verb bias or the underlying syntactic structure would influence our interpretation of the results. Given the high degree of similarity between the IWA and controls, it is unlikely that these variables significantly affected our results. Nonetheless, future studies might directly compare different verb classes and other sentence structures.

Finally, our data inherited nuances specific to AphasiaBank. The participant population of IWA necessarily had to perform some, if not all, of the language tasks for AphasiaBank. There may be an underrepresentation of IWA with more severe language deficits or other aphasia types that limit productive language abilities. More severely impaired IWA could 
show greater changes in their transitivity biases than the IWA included in our sample of AphasiaBank data.

\section{Conclusion}

This study characterized the presence and nature of transitivity biases in IWA's spontaneous speech, as recorded in structured interviews from AphasiaBank. Both controls and IWA exhibited transitivity biases in the same direction as normative verb biases from Gahl et al. (2004). There were no significant differences between IWA and controls' proportion of transitive or intransitive uses of the verb types, indicating that IWA retained transitive and intransitive verb biases. Overall, IWA (like controls) produced more utterances with intransitively biased verbs than with transitively biased verbs. The preference for intransitively biased verbs was greater for IWA. It may be that this verb preference reflects IWA's sensitivity to verb complexity (Kim \& Thompson, 2000), but this possibility will have to be further explored in future studies analyzing a range of verb complexities.

IWA's error rates were related to transitivity bias; mismatched productions (between transitivity bias and sentence structure) had significantly higher error rates than matched productions. Of the mismatched conditions, IWA produced more errors when using intransitively biased verbs in transitive structures. This finding further supports the possibility of an intransitive preference. Thus, the data suggest that IWA retain typical simple transitive and intransitive verb biases, and demonstrate sensitivity to verb complexity in their expressive language. These initial characterizations of IWA's transitivity biases are consistent with existing literature in receptive domains. In future corpus-based studies, we want to consider differently biased verbs, further our understanding of verb complexity as it relates to IWA, and continue refining our approaches to labeling IWA's ungrammatical speech.

\section{Supplementary Material}

Refer to Web version on PubMed Central for supplementary material.

\section{Acknowledgments}

We would like to thank the AphasiaBank consortium for providing the database for this research. We would also like to thank members of the Undergraduate Biology Research Program (UBRP) and the Speech Language and Brain Lab for their support. This work was supported by the HHMI under Grant 520006942 to the Undergraduate Biology Research Program at the University of Arizona and NIDCD Grant K23DC010808.

\section{References}

American Speech-Language-Hearing Association. Aphasia: Incidence and Prevalence. n.d

Basso A, Razzano C, Faglioni P, Zanobio ME. Confrontation naming, picture description and action naming in aphasic patients. Aphasiology. 1990; 4:185-195. DOI: 10.1080/02687039008249069

Berndt RS, Haendiges AN, Mitchum CC, Sandson J. Verb retrieval in aphasia. 2. Relationship to sentence processing. Brain and Language. 1997a; 56:107-137. DOI: 10.1006/brln.1997.1728 [PubMed: 8994700]

Berndt RS, Mitchum CC, Haendiges AN, Sandson J. Verb retrieval in aphasia. 1. Characterizing single word impairments. Brain and Language. 1997b; 56:68-106. DOI: 10.1006/brln.1997.1727 [PubMed: 8994699] 
Darwin CM, Gray LS. Going After the Phrasal Verb: An Alternative Approach to Classification. TESOL Quarterly. 1999; 33(1):65-83. doi:0.2307/3588191.

DeDe G. Lexical and prosodic effects on syntactic ambiguity resolution in aphasia. Journal of Psycholinguistic Research. 2012; 41(5):387-408. [PubMed: 22143353]

DeDe G. Effects of verb bias and syntactic ambiguity on reading in people with aphasia. Aphasiology. 2013a; 27:326-343. [PubMed: 23554543]

DeDe G. Verb transitivity bias affects on-line sentence reading in people with aphasia. Aphasiology. 2013b; 27(3):326-343. [PubMed: 23554543]

Engelter ST, Gostynski M, Papa S, Frei M, Born C, Ajdacic-Gross V, Gutzwiller F, Lyrer PA. Epidemiology of Aphasia Attributable to First Ischemic Stroke: Incidence, Severity, Fluency, Etiology, and Thrombolysis. Stroke. 2006; 37:1379-1384. [PubMed: 16690899]

Gahl S, Jurafsky D, Roland D. Verb subcategorization frequencies: American English corpus data, methodological studies, and cross-corpus comparisons. Behavior Research Methods, Instruments, \& Computers. 2004; 36(3):432-443.

Gahl S. Lexical biases in aphasic sentence comprehension: An experimental and corpus linguistic study. Aphasiology. 2002; 16:1173-1198.

Garnsey SM, Pearlmutter NJ, Myers E, Lotocky MA. The Contributions of Verb Bias and Plausibility to the Comprehension of Temporarily Ambiguous Sentences. Journal of Memory and Language. 1997; 37:58-93.

Kim M, Thompson CK. Patterns of comprehension and production of nouns and verbs in agrammatism: Implications for lexical organization. Brain and Language. 2000; 71:1-25. [PubMed: 10716790]

Lee J, Thompson CK. Agrammatic aphasic production and comprehension of unaccusative verbs in sentence contexts. Journal of Neurolinguistics. 2004; 17(4):315-330. [PubMed: 21311719]

Lee J, Thompson CK. Phonological facilitation effects on naming latencies and viewing times during noun and verb naming in agrammatic and anomic aphasia. Aphasiology. 2015; 29:1164-1188. DOI: 10.1080/02687038.2015.1035225 [PubMed: 26412922]

MacWhinney B, Fromm D, Forbes M, Holland A. AphasiaBank: Methods for studying discourse. Aphasiology. 2011; 25:1286-1307. [PubMed: 22923879]

MacWhinney, B. The CHILDES Project: Tools for Analyzing Talk. 3rd. Mahwah, NJ: Lawrence Erlbaum Associates; 2000.

Miceli G, Silveri MC, Villa G, Caramazza A. On the basis for the agrammatic's difficulty in producing main verbs. Cortex. 1984; 20:207-220. DOI: 10.1016/S0010-9452(84)80038-6 [PubMed: 6204813]

Roland D, Dick F, Elman JL. Frequency of basic English grammatical structures: A corpus analysis. Journal of Memory and Language. 2007; 57(3):348-379. [PubMed: 19668599]

Saffran EM, Berndt RS, Schwartz MF. The Quantitative Analysis of Agrammatic Production: Procedure and Data. Brain and Language. 1989; 37:440-479. [PubMed: 2804622]

Staub A. The Parser Doesn't Ignore Intransitivity, After All. Journal of Experimental Psychology: Learning, Memory, and Cognition. 2007; 33(3):550-569.

Thompson CK. Unaccusative verb production in agrammatic aphasia: the argument structure complexity hypothesis. Journal of Neurolinguistics. 2003; 16(2-3):151-167. [PubMed: 21274410]

Webster J, Franklin S, Howard D. An analysis of thematic and phrasal structure in people with aphasia: What more can we learn from the story of Cinderella? Journal of Neurolinguistics. 2007; 20:363394.

Whitworth A. Characterizing thematic role assignment in aphasic sentence production - Procedures for elicited and spontaneous output. European Journal of Disorders of Communication. 1995; 20(3):384-399. 
Individuals with Aphasia: Uses of Transitive Verbs

Controls: Uses of Transitive Verbs
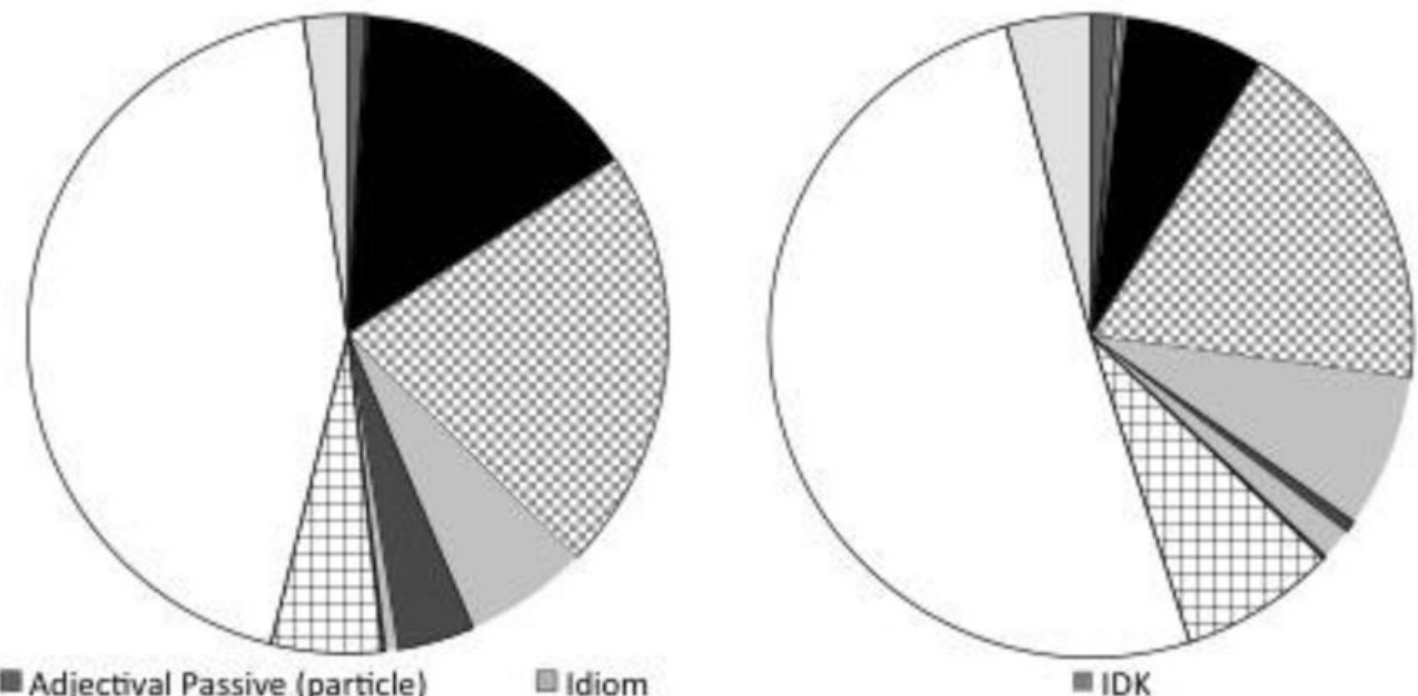

Adjectival Passive (particle)

DIdiom

mIDK

- Intransitive (particle)

B Infinitive

NP Complement

Other

III Passives (particle)

Quote (particle)

ESentential Complement (particle) $\square$ Transitive (particle)

EError in transcription

Figure 1.

IWA's and Controls' Uses of Transitively Biased Verbs in Various Sentential Structures.

Categories followed by (particle) indicate that forms with and without particles are collapsed in the analyses. 
Individuals with Aphasia: Uses of Intransitive Verbs

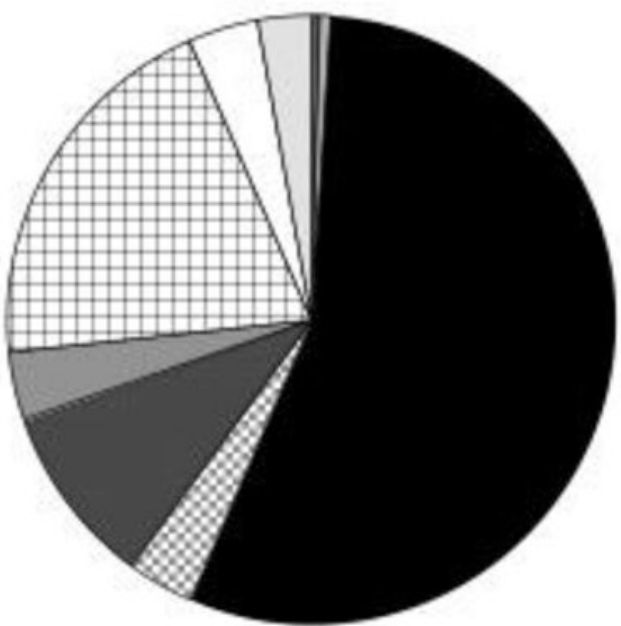

Adjectival Passive (particle)

- Intransitive (particle)

- other

דSentential Complement (particle)
DIdiom

Infinitive

= Passives (particle)

$\square$ Transitive (particle)

\section{Controls: Uses of Intransitive Verbs}

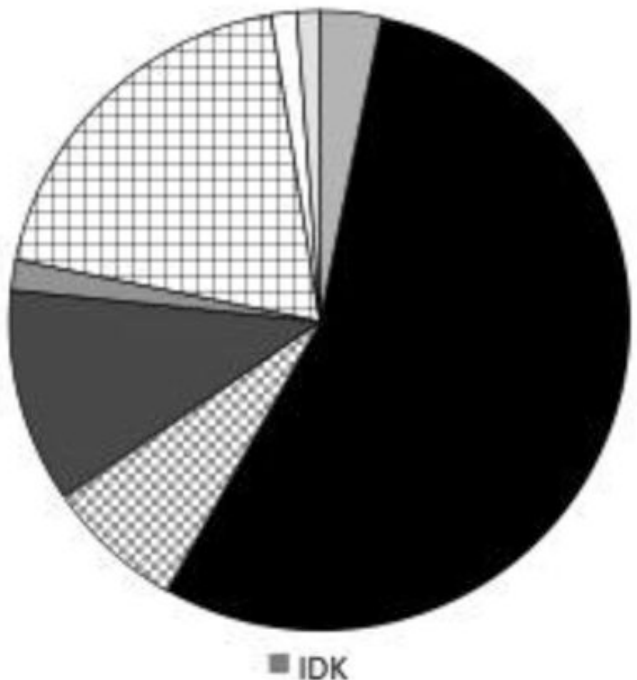

N NP Complement

Quote (particle)

$\square$ Error in transcription

Figure 2.

IWA's and Controls' Uses of Intransitively Biased Verbs in Various Sentential Structures. Categories followed by (particle) indicate that forms with and without particles are collapsed in the analyses. 


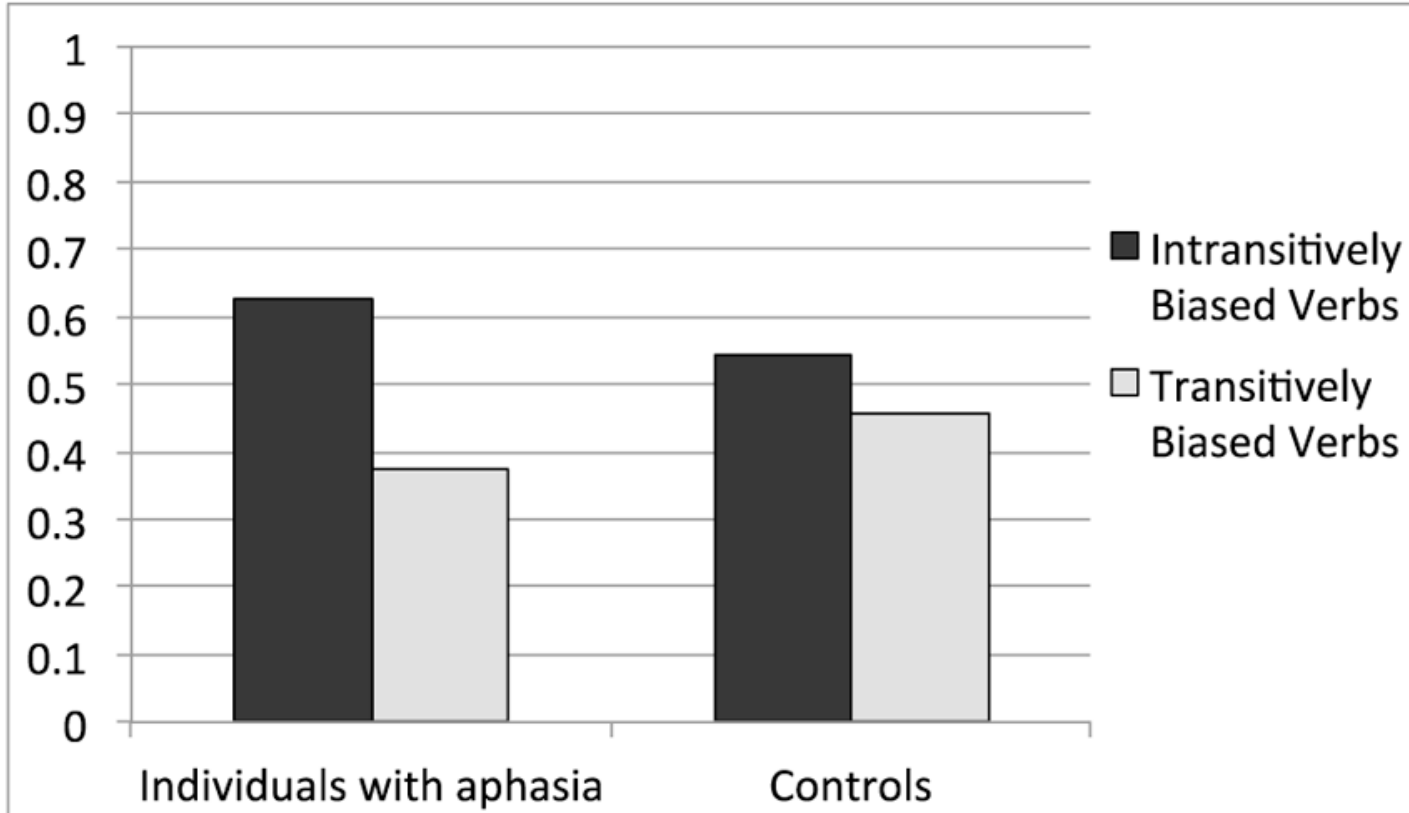

Figure 3.

IWA's and Control's Production of Transitively and Intransitively Biased Verbs (Regardless of Sentential Structure) 


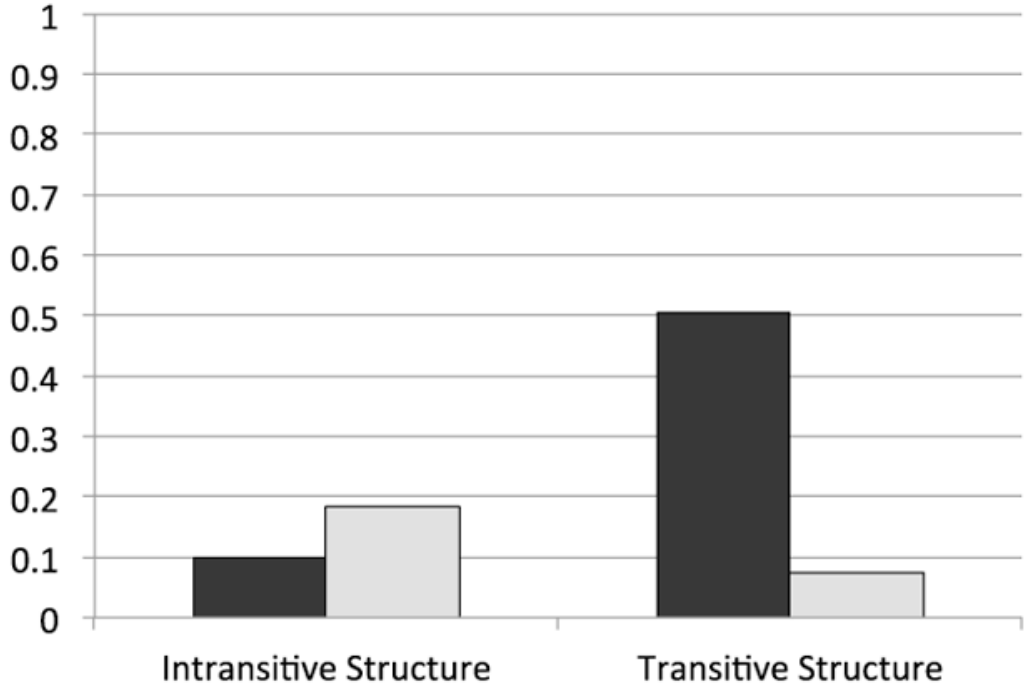

Figure 4.

Proportion of Utterances with Errors for Individuals with Aphasia
- Intransitively Biased Verbs $\square$ Transitively Biased Verbs 


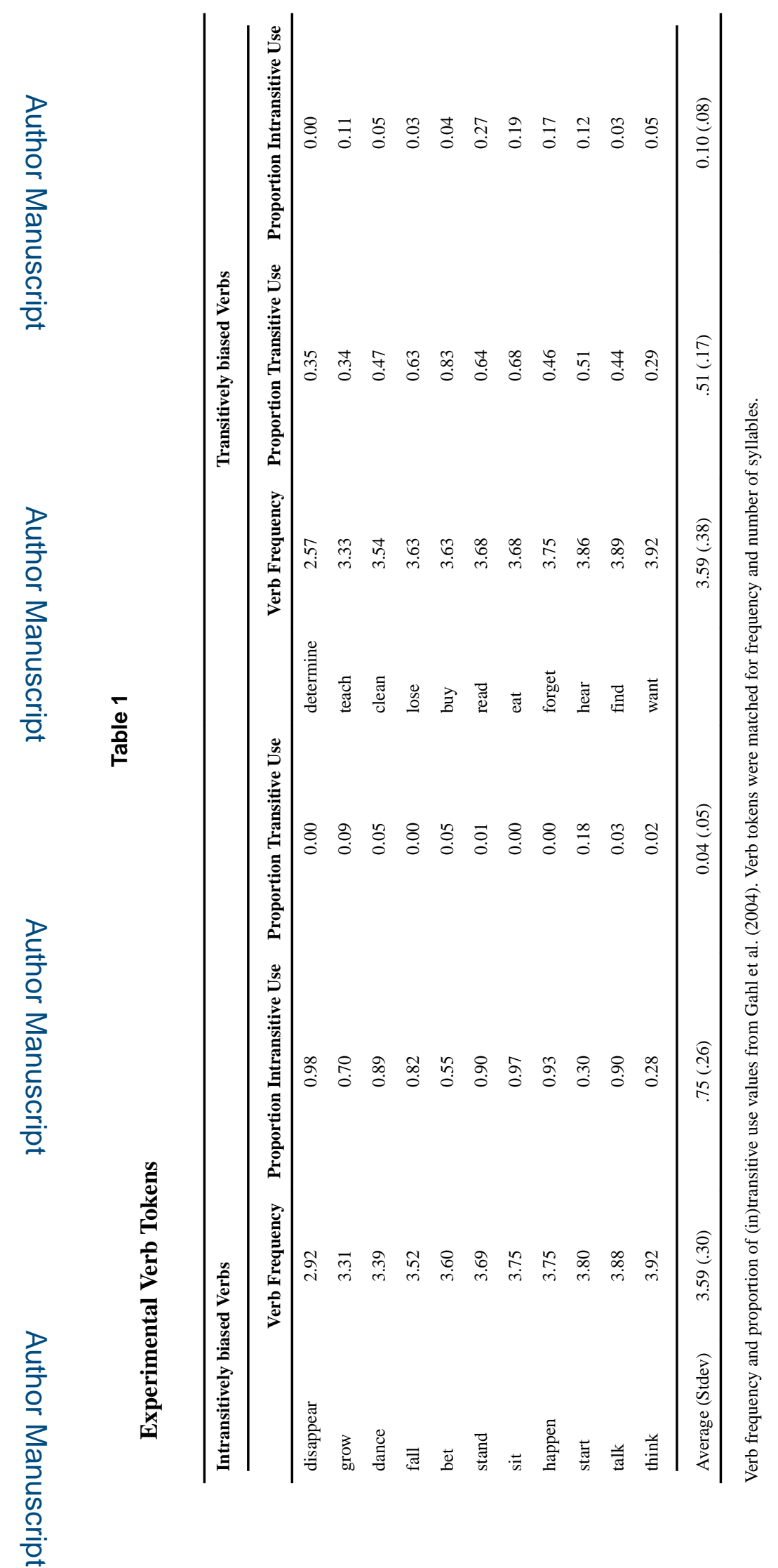

Aphasiology. Author manuscript; available in PMC 2018 April 30. 


\section{Table 2}

Verb Codes with Sentence Examples

\begin{tabular}{|c|c|c|}
\hline Error Code & Description & Examples \\
\hline$* \operatorname{tr}$ & Active transitive & I <bought $>$ the book. \\
\hline$* \operatorname{trpt}$ & Active transitive with particle & They <tried $>$ the slippers on. \\
\hline *in & Active intransitive & I can't $\langle$ talk $>$. \\
\hline *inpt & Active intransitive with particle & The guy's <standing> up. \\
\hline pass & Passive & I think that's what they're <called>. \\
\hline passpt & Passive with particle & I get < kicked> out. \\
\hline apass & Adjectival passive & He is <startled $>$. \\
\hline apasspt & Adjectival passive with particle & She acts < grown> up and she's not. \\
\hline sfin & Sentential complement & I don't <know> if I'm right or wrong. \\
\hline sfinpt & Sentential complement with particle & I < found $>$ out that, like, I was conscious. \\
\hline quote & Quotation & $\begin{array}{l}\text { I'm always surprise when people <tell > me, "Gee we had no idea there was } \\
\text { anything wrong with you talking." }\end{array}$ \\
\hline npcomp & Noun phrase complements & They <wanted> him to do something. \\
\hline passcomp & $\begin{array}{l}\text { Passives with sentential or VP } \\
\text { complements }\end{array}$ & I was <taught> how to listen, how to watch. \\
\hline $\inf$ & Infinitive complements & $\begin{array}{l}\text { So she was trying to run away from this beautiful man that <wanted> to marry } \\
\text { her. }\end{array}$ \\
\hline other & Everything else & I don't <know>. I <remember> going to work with an accountant. \\
\hline $\mathrm{x}$ & Typos in transcription & Just prior to my stroke I was taking a < driving > trip by myself. \\
\hline $\mathrm{i}$ & Idioms & $\mathrm{He}<$ fell $>$ in love with her and got married. \\
\hline
\end{tabular}

Note: Coding scheme was adapted from Gahl et al., 2004. Coded verbs are indicated by brackets. Codes that indicated transitive or intransitive frames are noted with an asterisk. 
Table 3

Error Coding Scheme with Examples.

\begin{tabular}{|c|c|c|}
\hline Error Code & Description & Example \\
\hline lbv & Lexical error before verb & I nont $<$ know $>$ how she got the $\ldots$ this... \\
\hline lav & Lexical error after verb & because they don't <want> your faudis. \\
\hline omfb & Omission of function word before verb (including auxiliary verbs) & Then the guy... you know, <dancing > and all of that... \\
\hline omfa & Omission of function word after verb & $\ldots$ and they $<$ drove $>$ her the party. \\
\hline $\operatorname{minf}$ & Morphological/inflectional error on the verb itself & The cat is just <sit> there. \\
\hline ebv & Error before verb (non-lexical) & I went any time 〈tried〉. \\
\hline eav & Error after verb (non-lexical) & I $<$ think $>$ is talking. \\
\hline $\mathrm{pp}$ & Phonological paraphasia & I <sot> something was wrong with my arm. \\
\hline aband & Abandoned utterance & I don't $<$ know $>$ what made Cinderella... \\
\hline NR & Not relevant & Mother was dead but someone < thought $>$ it and was in \\
\hline
\end{tabular}

Note: Coded verbs are indicated by brackets. 